\title{
AIDS-Related Kaposi Sarcoma T1 (Poor Risk): Other Non-Nodal Viscera Involvement
}

National Cancer Institute

\section{Source}

National Cancer Institute. AIDS-Related Kaposi Sarcoma T1 (Poor Risk): Other Non-

Nodal Viscera Involvement. NCI Thesaurus. Code C134977.

AIDS-related Kaposi sarcoma involving organs other than the lungs or gastrointestinal tract. It includes liver, bladder, reproductive tract, heart, and endocrine organs. 